\title{
The seeds of cosmic structures as a door to Quantum Gravity Phenomena
}

\author{
Daniel Sudarsky ${ }^{* \dagger}$ \\ Instituto de Ciencias Nucleares UNAM \\ E-mail: sudarsky@nucleares.unam.mx
}

This paper contains a critique of the standard inflationary account of the origin of cosmological structures from quantum fluctuations in the early universe. This critique can be thought to be purely philosophical in nature, but I prefer to view it, rather, as arising from the need to put the interpretational aspects of the theory -which quite obviously lie at the basis of any comparison with experiments- on the firm grounds required by the unique features of the problem at hand. This discussion is followed by a proposal to complement that treatment to deal with the unsatisfactory aspects of the standard account of the problem, using Penrose's ideas about the quantum gravity induced collapse of the quantum states of matter fields. The formalism developed to carry out this analysis was first introduced in (1) and leads to unexpected predictions and to novel avenues to confront some of the details of the proposal with observations. In my view, this is, therefore, the most promising path towards quantum gravity phenomenology.

From Quantum to Emergent Gravity: Theory and Phenomenology

June 11-15 2007

Trieste, Italy

* Speaker.

$\dagger$ A footnote may follow. 


\section{The Problem}

The inflationary theory of the origin of structure in our universe, although generally acclaimed for its successes $(2 ; 3)$, has a very unsettling aspect: it does not account for the transition from a homogeneous and isotropic (H.\&I.) early state of the universe to its late, anisotropic and in-homogeneous state. What do we mean by that? Isn't this, precisely, what the account we read in books and articles does in calculating the spectrum of primordial fluctuations? We will see that the answer one gives to this question depends very strongly on what one expects a physical theory to be able to deliver, an issue which is strongly correlated with one's views about what quantum mechanics is and what it is not. The noteworthy point is, however, that despite what it might seem at first sight, the way these issues are approached have implications that transcend the purely philosophical aspects and impinge on our understanding of one of the fundamental questions in modern cosmology. But let us go back to the questions raised: does the standard inflationary scenario, and the accompanying calculations of the spectrum of primordial fluctuations, really account for the transition from a H.\&I. early state of the universe to its late, anisotropic and inhomogeneous state ${ }^{1}$ ? If the answer to the above question depends on one's view of quantum theory, why does this article start with such a categorical assertion, that the answer is in the negative? My point will be that the views that one would be led to take about quantum mechanics, in seeking a justification to answer in the positive, are not one that physicists would like to take, are not one that would justify its use in the situation at hand, and/or are not one that would allow one to consider the sort of issues raised in the search for quantum gravity.

This article will be devoted, to a large extent, to deal with the issues above, but will include a brief description of the richness in perspective that can be achieved if one takes a stricter ontological view of the essence of quantum physics. We will see that not only will we gain the right to ask more profound questions, but that, in attempting to deal with them, we will be led to new and surprising insights about the nature of quantum gravity and their relevance to the understanding of the early universe. The fact that issues that can initially be deemed to be merely philosophical, can transcend such domain and enter the realm of observational scientific inquire, can be expected to come as a shock to people belonging to the generations of physicists trained not to ask that type of questions. However, these questions are indeed some of the hardest ones, and it is the hope of this author that this manuscript may contribute to the changing of the above mentioned unfortunate trend.

\section{What is quantum physics?}

It must be surely pretentious beyond belief to raise this issue in the company of prestigious physicists that have devoted their lives to the even more complex problem of making gravitation and quantum physics compatible. These efforts have been concentrated in the highly nontrivial development of the mathematical language and the subtleties that arise in constructing plausible theoret-

\footnotetext{
${ }^{1}$ This point is sometimes characterized as the "transition from the quantum regime to the classical regime", but I find this a bit misleading: most people would agree that there are no classical or quantum regimes. The fundamental description ought to be quantum always, but there are regimes in which certain quantities can be described to a sufficient accuracy by their classical counterparts representing the corresponding expectation values. This depends of course on the physical state, the underlying dynamics, the quantity of interest, and the context in which we might want to use it.
} 
ical models applicable to quantum gravity. Perhaps, it is the enormity of the task and the difficulty of that challenge, that having absorbed the attention of the most brilliant minds in our field, have left as relatively much less explored the issues which I will be rising.

These are related to the so called "measurement problem in quantum mechanics" a subject that has puzzled physicists and philosophers of physics from the time of the inception of the theory (4). We will however focus here on one particular instance of of the problem: the cosmological setting, which is a subject that has received much less attention from the physics community. There are of course notable exceptions to the assessment above, represented by thinkers like R. Penrose, (5) J. Hartle (6), and others who have faced these and related issues with very open and critical minds. Having said all this, I must nevertheless try to answer the question posed, if for no other reason, just to make my position clear to the reader, and help him understand the hows and whys of the postures taken in this manuscript. To do this I will go through some of the postures that I have encountered on the subject:

a) Quantum physics as a complicated theory of statistical physics. By this, I am referring to the position that holds that quantum mechanics acquires meaning only as it is applied to an ensemble of identical systems. In this view, one must accept that a single atom in isolation is not described by quantum mechanics. Let us not get confused by the correct but simply distracting argument that atoms in isolation do not exist. The point is whether, to the extent to which we do neglect its interactions with distant atoms, and specially with the electromagnetic field which, even in its vacuum state is known to interact with the atom, quantum mechanics is applicable to the description of a single atom. Again, what can we mean by that, if we know that in order to be able to say anything about the atom, we must make it interact with a measuring device? Well, the question is simply whether applying the formalism of quantum mechanics to treat the isolated atom can be expected to yield correct results as it pertains the subsequent measurements.? One might think that this is a nonsensical question, as these results are always statistical in nature. The point is that this statement is not really accurate: for instance if the atom (say, of hydrogen) was known to having been prepared in its ground state, the probability of measuring any energy other than the one in the ground state is zero. Furthermore, the probability of observing the atom in a high angular momentum eigenstate is equally zero. In fact, for any observable commuting with the hamiltonian the predictions are not statistical at all, but $100 \%$ deterministic and precise! If so, there must be something to the description of that single atom by its usual quantum mechanical state, and thus it becomes blatantly false the notion that quantum mechanics can not be applied to single system. What is true, of course, is that, in applying the theory to a single system, the predictions we can make with certainty are very limited, with the extent of such limitations being determined both by the nature of the system's dynamics, and by the way the system was initially prepared. Moreover, in relation with the issue that concerns us in this article, taking a posture like this, about quantum physics, would be admitting from the beginning that we have no right to employ such theory in addressing questions concerning the unique universe to which we have access, even if we were to accept that somehow there exists an ensemble of universes to which we have no access. Note, moreover, that we should beware from confusing statistics of universes and statistics within one universe. Furthermore, if a quantum state serves only to represent an ensemble, how is each element of the ensemble to be described? Perhaps, it can not be described at all ? What do we do in that case with our universe? 
b) Quantum physics as a theory of human knowledge. Within this view of quantum theory, the state of a quantum system does not reflect something about the system, but just what we know about the system. Such view, naturally rises the question what is there to be known about the system if not something that pertains to the system? The answer comes in the form of: correlations between the system and the measuring devices, but then, what is the meaning of these correlations? The usual meaning of the word correlation implies some sort of coincidence of certain conditions pertaining to one object with some other conditions pertaining to the second object. However, if a quantum state describes such correlation, there must be some meaning to the conditions pertaining to each one of the objects. Are not these, then, the aspects that are described by the quantum mechanical state for the object? If we answer in the negative, it must mean that there are further descriptions of the object that can not be casted in the quantum mechanical state vector. On the other hand, if we answer in the positive we are again taking a view whereby the state vector says something about the object in itself. Perhaps we are just going in circles. For those who read these considerations as philosophical nonsense, let us just say that if we follow this view, we have no right to consider questions about the evolution of the universe in the absence of sapient beings, and much less to consider the emergence, in that universe, of the conditions that are necessary for the eventual evolution of humans, while using a quantum theory. I would take it even further: what would be the justification for considering states in any model of quantum gravity, if we took such view of quantum physics?

c) Quantum physics as an non-completable description of the world. With this I am referring to any posture that effectively, if not explicitly, states:"The theory is incomplete, and no complete theory containing it exists or will ever do" . This view will be considered as being held by the many physicists that, while not openly advocating such posture, will direct us to use quantum mechanics " as we all know how " while reminding us with a stern voice that no violation of quantum mechanics has ever been observed. While this is with not doubt a literally correct statement, we must remind our colleagues that by this, they refer, of course, to the rules as found in any quantum mechanics text book, that essentially rely on to the Copenhagen interpretation, which as we all know, raises severe interpretational issues that become insurmountable once we leave the laboratory and consider applying quantum theory to something like the universe itself. According to this view, we should content ourselves using its tools, and making, in the situation at hand, non-rigorous predictions $^{2}$. We must acknowledge, however, that in situations where one can not point to the classical-quantum dividing line, where we can not identify the system and the apparatus, nor the observables that are to be measured, the entity carrying out those measurements and the time at which the measurements are to be thought as taking place, we have, in fact, no clearly defined scheme specifying how to make the desired predictions. That is, in dealing with the questions pertaining to the early universe in terms of quantum theory, we have no clear and specific rules for making predictions. However, according to the colleagues arguing for such practical posture, we should be content with the fact that the predictions have in fact been made, and that they do seem to agree with observations. The issue is, of course, that in the absence of a well defined set of rules,

\footnotetext{
${ }^{2}$ Quantum theory gives perfectly well defined and rigorous predictions, which are in general of a statistical nature, once we have identified the state of the system the observable that is going to be measured, which must include in general the time at which the measurement occurs. The in the standard treatments of the situation at hand no such explicit identifications are made and thus the line of reasoning connecting the formalism to the predictions is best described as non-rigorous.
} 
that are explicit to the point where a computer could, in principle, arrive to the predictions using only the explicit algorithm and the explicitly stated inputs, we have no way to ascertain whether or not, such "predictions" do or do not, follow from the theory. We can not be sure whether or not some unjustified choices, manipulations and arguments have been used as part of the process by which the predictions have been obtained. Correct predictions are not enough ${ }^{3}$ ! They must first be actual predictions. This is quite clear, particularly, when the argumentative connections used in arriving to the predictions are so loose that no one can be sure whether these are in fact predictions or not. Specially suspect are, of course, those "predictions" that are, in fact, retrodictions, and on this point we should be aware that long before inflation was invented, Harrison and Zel'dovich (7) had already concluded the form of the primordial spectrum, based on rather broad observations about the nature of the large scale structure of our universe.

d) Quantum physics as part of a more complete description of the world. Here we are not referring to an extension of the theory into some sort of hidden variable type, as the problem we want to rid the theory from is not its indeterminism, but the so called measurement problem. Completing the theory would require something that removes the need for a external measurement apparatus, an external observer, etc. There are, for instance, ideas like generalization of quantum physics using a scheme based on sums over decoherent histories proposed by J. Hartle (6), others invoking something like the dynamical reduction models proposed by Ghirardi, Rimini \& Weber (8), and the ideas of R. Penrose about the role of gravitation in modifying quantum mechanics in the merging of the two aspects of physical reality (5). The present manuscript, as well as the original treatment of these issues carried out in collaboration with A. Perez and H. Shalmann (1), are inspired on Penrose's generic ideas.

e) Quantum physics as a complete description of the world. The view that quantum mechanics faces no open issues and that, in particular, the measurement problem has been solved. Among the holders of these views one can further identify two main currents: those that subscribe to ideas along the so called "many world interpretation of quantum mechanics" and consider this to be a solution to the measurement problem, and those that hold a view that the measurement problem in quantum mechanics has been solved by the consideration of "decoherence". Let us first note that the many world interpretation does very little to ameliorate the measurement problem, as there is a mapping between what in that approach would be called the splittings of worlds, and what would be call "measurements" in the Copenhagen interpretation. Thus every question that can be made in the latter interpretation has a corresponding one in the many worlds interpretation. For the case of the measurement problem the issues would be. when does a world splitting occur? why, and under what circumstances does it occur? What constitutes a trigger? Concerning the decoherence mechanism as a solution to the measurement problem I would like to start by quoting the postures

\footnotetext{
${ }^{3}$ For those that shiver upon the last statement let me recall a theory about venereal diseases that was popular in ancient Rome: venereal diseases come from Venus, the Goddess of Love, obviously. The celestial enemy of Venus was Mercury, which constantly challenged the Goddess's for the attention of the Sun (Apollo). Fortunately for humans, Mercury had a substance associated with him, that could be found on Earth: the metal mercury. It was then evident that substances containing mercury should help in fighting the diseases associated with Venus. And it was a known fact (at least among the practitioners of medicine) that certain mercury salts did provide substantial relief for those infected with the most common of the venereal diseases of the time. I do not, of course, pretend to compare any theory of modern physics with ancient Rome's medical arguments. My point is only to illustrate the statement I made above: correct predictions are not enough.
} 
that in these regards are held by several people that have considered the issue at length in order to dispel the widespread notion that such is the consensus view:

"Many physicist nowadays think that decoherence provides a fully satisfying answer to the measurement problem. But this is an illusion." Arnold Neumaier (9).

"...note that the formal identification of the reduced density matrix with a mixed state density matrix is easily misinterpreted as implying that the state of the system can be viewed as mixed too.. .. the total composite system is still described by a superposition, it follows from the rules of quantum mechanics that no individual definite state can be attributed to one of (the parts) of the system ...", M. Schlosshauer (10).

"Does decoherence solve the measurement problem? Clearly not. What decoherence tells us is that certain objects appear classical when observed, But what is an observation? At some stage we still have to apply the usual probability rules of Quantum Theory." E. Joos (11).

In fact, we will see that when dealing with cosmology the problem becomes even more vexing and acute. Nonetheless, most researchers in the field seem to take some version of decoherence as the paradigm where the direct application of the standard forms of quantum mechanics to the problem at hand finds its justification. Significantly, the diversity of precise approaches indicates some degree of in-satisfaction of some researchers with the views of others (12).

Before engaging on the cosmological case, let us review briefly what decoherence is, and what can and can not do.

Decoherence is the process by which a system that is not isolated, but in interaction with an environment (as are all physical systems except the universe itself) "looses" or " transfers" coherence into the degrees of freedom of such environment. It is a well studied effect that follows rather than supersedes the laws of quantum physics. It is, therefore, clear that, in principle, it can not be thought to offer explanations that go beyond what can be directly inferred from the application of the principles of quantum physics. Its main achievement is to allow for the studying the conditions in which the quantum interferences expected from the idealized consideration of a system as isolated, become observationally suppressed as the result of the system's interaction with the environment.

The basic recipe for an analysis of decoherence in a given situation follows the following steps:

1) Divide D.O.F. : system + environment ( identify inaccessible or irrelevant D.O.F.).

2) Compute Reduced Density matrix (trace over environment D.O.F.).

3) Perform suitable time average so that the off-diagonal matrix elements vanish.

4) Regard the diagonal density matrix as describing a statistical ensemble.

The Problems: once one has understood why certain interferences can not be observed in practice, it is tempting to conclude that one has understood the "emergence of classicality", and that therefore there is nothing left of the so called " measurement problem" in quantum mechanics. This turns out to be a simplistic and misguided conclusion, as indicated by the quotations listed above. There are in fact, at least two very serious problems with considering decoherence in this light:

I) The basis problem: it is clear that the diagonal nature of the reduced density matrix obtained in the step 3) of the program above, will be lost, in general, upon a change of basis for the Hilbert space of the system at hand. This is taken to mean that the nature of the system-environment interaction selects a so called pointer basis which underscores the aspects that have become classical as a result of decoherence. The point of course is that this leaves one with the usual situation whereby, if the 
selected basis is, say, the position basis, the momentum of the system remains undetermined and thus one can not argue that classicality has really emerged.

II)The definite outcomes problem: here the problem is the absence of sufficient justification for the interpretation of the mixed state described by the density matrix as describing a statistical ensemble and in regarding a single system as being in definite yet unknown state among the ones represented in the diagonal elements of the density matrix. The result that emerges from the decoherence calculations rather indicates that the system must be regarded as coexisting in the various alternatives, but where the interferences in the observables associated with the particular pointer basis being suppressed. Selecting among these alternatives can be viewed as deciding between the " choice vs coexistence" interpretations. In order to argue that decoherence really leads to the emergence of classicality one would have to advocate the "choice" interpretation, but well known, and experimentally confirmed aspects of quantum mechanics such as the violation of Bell inequalities, force us to opt for the "coexistence" interpretation $(14 ; 15)$.

The next example, from ordinary non-relativistic quantum mechanics, serves as a clear analogy of the situation we face: consider a single particle in a state corresponding to a minimal wave packet centered at $\vec{X}=(D, 0,0)$ ( the vectors in 3-D space are given in cartesian coordinates (x, y, z)). Let the particle have its spin pointing in the $+y$ direction. Take this state and rotate it by and angle $\pi$ about the $z$ axis. Now consider the superposition of the initial and the rotated states. The resulting state is clearly symmetric under rotations by $\pi$ around $z$. Now consider taking the trace over the spin D.O.F. The resulting density matrix is diagonal. Can we say that the situation has become classical? Of course not. Is the state still invariant under rotations of magnitude $\pi$ about the $z$ axis? Obviously yes. Can a mathematical manipulation with no physical process counterpart ever change the state of the system? Answering yes would take you to the view discussed in b).

\section{The exacerbated problem: applying quantum physics to the early universe}

We should point out that some researchers in the field, such as (16), have acknowledged that there is something mysterious in the standard account of the emergence of structure, and people like $\mathrm{J}$. Hartle (6) that have pointed out the need to generalize quantum mechanics to deal with cosmology, and of course R. Penrose, who in his last book (17) has stressed the relevance of the general measurement problem in quantum mechanics to the problem of breakdown of the H.\&I. during inflation, comparing it with the problem of the breakdown of spherical symmetry in a particle decay. In my view, this analogy does not emphasize the point that, in the cosmological context, the problem is even more severe than in ordinary situations, because, in that case, we can not even rely on the strict Copenhagen interpretation as a source of safe practical rules.

As an example that exhibits quite clearly the deepening of the problem in this context, let's consider the following quotation from a well known thinker on these sort of issues in quantum theory: "As long as we remain within the realm of mere predictions concerning what we shall observe (i.e. what will appear to us) and refrain from stating anything concerning "things as they must be before we observe them " no break in the linearity of quantum dynamics is necessary. " D'Espagnat (18).

However, in the cosmological setting, we need to deal precisely with this situation: we need to think about the state of affairs of the universe before the emergence of the conditions 


\section{that make us possible, before we existed and before we ever carried out an observation or measurement.}

In the cosmological setting, we seek a historical, that is a time development, description of cosmic evolution that follows the laws of physics (would this be, perhaps, too much to ask of a physical theory of cosmology?). Such description should explain how did WE arise, in a path covering the emergence of the primordial density fluctuations, of galaxies, stars, and planets, and eventually living organisms, humans, cultures, etc.. Such an account should not rely on the measurement (in) abilities of the late evolved creatures to explain the emergence of conditions that make them possible. From this perspective, one can not justify identifying some D.O.F as irrelevant environment, based on the current, or even permanent, limitations of humans, in the analysis of the emergence of the primordial density fluctuations, for doing so leads to a circular argument with no explanatory value.

Furthermore, one might be asked, when attempting to follow some of the standard versions of the explanations of these delicate points, to accept one or more of the following notions: 1)that quantum physics does not describe our universe, as it was never H.\&I., only a certain ensemble of universes was. The argument here seems to rely on the notion of such ensemble of universes could be used to describe aspects of our universe that could not be described in isolation. The line of thought would indicate that it is the superposition of the states of all the universes in the ensemble, what is represented by the H.\&I. quantum state. The problem is that this is not quantum mechanics: if we have two systems, each of which is described by a quantum state, the composite system is not described by the superposition of the two quantum states (it is described by their direct or "tensor" product in the direct product of the corresponding Hilbert spaces). 2) that our universe is still H.\&I., and that the appearances to the contrary are the result of our inability to observe all the degrees of freedom. This posture makes us and our limitations, a fundamental part of the explanation of the origin of the conditions that makes our existence possible. 3) That "it does not matter when the universe stopped being H.\&I. ", without being able to even address issues such as when?, why?, or due to what?.

I find it quite remarkable that many physicists seem to look for, or content themselves with, what I would call technical pseudo-answers to the problem. These approaches can be globally characterized as calculation that while technically correct fail to address the issue at hand by the implicit acceptance of an interpretational scheme of the formalism, which is not justified in the present context. These often include pseudo-analogies, that is, comparisons of the present problem with other problems which superficially seem complete analogies but that upon careful examination reveal that they are missing crucial aspects, particularly those that make the problem at hand such a challenge. The danger of the analysis' by analogy is that they often fail to dissect the problem to the point where all aspects of the analogy have been explicitly exposed and can therefore be examined. Consider, for instance, a recent article (19) which proposes an analogy between the process that lies at the origin of the anisotropies and inhomogeneities in the early universe, with the process of particle creation out of the vacuum in the presence of a sufficiently intense electric field, a process known as the Schwinger process (20). In that calculation, one evaluates the $S$ matrix element between the "in" vacuum and the "out" vacuum, and interprets the difference between the result and the unity as a measure of particle creation. This identification would be justified by unitarity and the observation that the $S$ matrix element between the "in" vacuum and the other vectors in the 
"out" Hilbert space, such as a specific state with a single pair of electron and positron, would be interpreted as the probability of pair creation in such state. This would in turn, be justified by the standard quantum mechanics postulate of the projection postulate, indicating that $|\langle A \mid B\rangle|^{2}$ as the probability of finding the system in the state $|A\rangle$, if it was originally prepared in the state $|B\rangle$. But this assumes that an observation, or measurement is carried out for an observable for which $|A\rangle$ is a (non-degenerate) eigenstate. In the absence of a measurement, the system must be in the state given by the unitary evolution of the state $|B\rangle$. The probability interpretation is only valid in conjunction with a measurement.

To see the problems that entail the extrapolation into unjustified realms of the standard interpretative scheme of quantum mechanics, let us consider in more detail the Schwinger process: we start by noting that we must imagine the electric field to be turned on at some finite time, for otherwise, the problem of the electron filed in interaction with an eternal electric filed, would be stationary, and if the system was prepared in its vacuum state, or the state corresponding to the minimal eigenvalue of the full hamiltonian, it would remain in that state for all times and the issue of pair creation would not make any sense. So let us assume that the electric field is turned on at some time $t_{1}$ ( one is often interested in considering such process to be carried out adiabatically, so we can imagine this taking place during a long interval $\Delta(t)$ centered at $\left.t_{1}\right)$. Analogously, we assume the electric field to be turned off at $t_{2}$. Let us assume that that electric field points in the $\mathrm{x}$ direction. We now assume that the system is prepared at a time $t<<t_{1}$ in the vacuum state $(|i n, 0\rangle)$ and we ask about the probability amplitude for observing the system in the state containing an electron and a positron, in the single particle states $\psi_{1}$ and $\psi_{2}$ respectively. This question has a very well defined answer in quantum theory, which is simply $\left\langle 0 ;\right.$ in $|S|\left(\psi_{1},-\right),\left(\psi_{2},+\right)$; out $\rangle$ (of course this might not be easy to compute but that is quite a different issue). However, let us note that we can not assume that the system, in the absence of a measurement, has a well defined probability of being in the state $\mid\left(\psi_{1},-\right),\left(\psi_{2},+\right) ;$ out $\rangle$, among other things, because the initial state is invariant under translations in the $y, z$ plane, the hamiltonian preserves this invariance but the state $\mid\left(\psi_{1},-\right),\left(\psi_{2},+\right)$; out $\rangle$ will not in general share such invariance. Thus, while we are perfectly justified in viewing the $\mathrm{S}$ matrix calculation to yield the prediction for probability for the observation of pair creation out of the vacuum when contemplating the measurement of the number of pairs at a certain time, we are not justified in regarding the state of a field as being anything but $U|B\rangle$ ( where $\mathrm{U}$ is the unitary evolution operator ) before a measurement is carried out. Similarly in the early universe inflationary context, we have no right to view the state of the universe as anything but $U|0\rangle$ in the absence of a measurement.

Again some of the most conspicuous and clear contradictions that arise when we give ourselves the right to use unjustified interpretational extrapolations, can be seen by considering the standard B-EPR(21) setup while entertaining the notion that the two particles might have a particular spin orientation in the absence (or before) a measurement is carried out (22). We could, for instance, decide to trace over the spin D.O.F. of one of the particles of the EPR pair and obtain, for the spin D.O.F. of the other particle, a diagonal density matrix, and be tempted to interpret this as indicating that the particle has one of the two spins orientations. But we know this leads to contradictions.

I'll try to convince the reader that this is not necessarily "just philosophy", and that the early universe offers the "Laboratory " where some of the issues can, at least in principle, be studied. It is worth pointing out that, in fact, the motivations for inflation itself are often criticized as being "too 
philosophical".

\section{What to do? Our approach.}

As we have seen, we need a paradigm that will allow us to consider a transition from a H.\&I. state for the universe to a state that is neither isotropic nor homogeneous, but without relying on an external system to carry out a measurement and without reliance on our own limitations as observers to select a set of degrees of freedom to be considered as unobservable, and so forth. Our approach will follow in this case the suggestions by Roger Penrose that quantum mechanical wave function collapse is an actual dynamical process where a system is forced to jump into one of a certain collection of states, breaking, in the process, the unitary evolution of quantum mechanics. Moreover, these ideas suggest that the fundamental theory of gravity ${ }^{4}$ might play a central role in the actual physics of collapse, by forbidding certain types of superpositions of gravitational degrees of freedom to exist for more than a very brief time. These ideas are certainly a bit too schematic to allow us to carry a detailed analysis of the issues at hand, so we will consider a concrete formalism inspired on them. The idea behind the scheme we will be using is that the quantum gravity requires both the modification of our theory of gravitation and that of quantum mechanics. In this scheme the fundamental degrees of gravitation are not related to the metric degrees of freedom in any simple way, but instead the latter appear as effective degrees of freedom of a non-quantum effective theory. The degrees of freedom of other fields whether or not fundamental are to be considered as susceptible to a quantum treatment on their own, an assumption that would avoid problems when contrasting this paradigm with the well established successes of quantum theory in nongravitational realms.

These ideas leave room to consider, therefore, situations where a quantum treatment of other fields would be appropriate but a classical treatment of gravitation would be justified. That is the realm of semi-classical gravity that we will assume to be valid for most of the time. However, this approximation would break down in association with the quantum mechanical jumps that are considered to be part of the underlying quantum theory containing gravitation.

In accordance with the ideas above we will use a semi-classical description of gravitation in interaction with quantum fields as reflected in the semi-classical Einstein's equation $R_{\mu v}-(1 / 2) g_{\mu \nu} R=$ $8 \pi G\left\langle T_{\mu v}\right\rangle$ whereas the other fields are treated in the standard quantum field theory (in curved space-time) fashion. This is supposed to hold at all times except when a quantum gravity induced collapse of the wave function occurs, at which time, the excitation of the fundamental quantum gravitational degrees of freedom must be taken into account, with the corresponding breakdown of the semi-classical approximation. The possible breakdown of the semi-classical approximation is formally represented by the presence of a term $Q_{\mu \nu}$ in the semi-classical Einstein's equation which is supposed to become nonzero only during the collapse of the quantum mechanical wave function of the matter fields. Thus we write

$$
R_{\mu v}-(1 / 2) g_{\mu v} R+Q_{\mu v}=8 \pi G\left\langle T_{\mu v}\right\rangle
$$

\footnotetext{
${ }^{4}$ Here the suggestion is that such theory is not just the result of the standard type of quantization as applied to gravity, but something involving a radical change in our paradigm of physical theories.
} 
Thus, we consider the development of the state of the universe during the time at which the seeds of structure emerge to be initially described by a H.\& I. state for the gravitational and matter D.O.F.. At some stage, the quantum state of the matter fields reaches a stage whereby the corresponding state for the gravitational D.O.F. is forbidden, and a quantum collapse of the matter field wave function is triggered. This new state of the matter fields does no longer need to share the symmetries of the initial state, and by its connection to the gravitational D.O.F. now accurately described by Einstein's semi-classical equation leads to a geometry that is no longer homogeneous and isotropic.

\section{The inflationary origin of cosmic structures: the amended story, or the story after the Gospel}

The starting point of the analysis is as usual the action of a scalar field coupled to gravity ${ }^{5}$

$$
S=\int d^{4} x \sqrt{-g}\left[\frac{1}{16 \pi G} R[g]-1 / 2 \nabla_{a} \phi \nabla_{b} \phi g^{a b}-V(\phi)\right],
$$

where $\phi$ stands for the inflaton and $V$ for the inflaton's potential. One then splits both, metric and scalar field into a spatially homogeneous ('background') part and an in-homogeneous part ('fluctuation'), i.e. $g=g_{0}+\delta g, \phi=\phi_{0}+\delta \phi$.

Th equations governing the background unperturbed Friedmann-Robertson universe with line element $d s^{2}=a(\eta)^{2}\left[-d \eta^{2}+\delta_{i j} d x^{i} d x^{j}\right]$, and the homogeneous scalar field $\phi_{0}(\eta)$ are, the scalar field equation,

$$
\ddot{\phi_{0}}+2 \frac{\dot{a}}{a} \dot{\phi}_{0}+a^{2} \partial_{\phi} V[\phi]=0,
$$

and Friedmann's equation

$$
3 \frac{\dot{a}^{2}}{a^{2}}=4 \pi G\left(\dot{\phi}_{0}^{2}+2 a^{2} V\left[\phi_{0}\right]\right) .
$$

The background solution corresponds to the standard inflationary cosmology which written using a conformal time, has, during the inflationary era, a scale factor $a(\eta)=-\frac{1}{H_{1} \eta}$, with $H_{I}^{2} \approx$ $(8 \pi / 3) G V$ and with the scalar $\phi_{0}$ field in the slow roll regime so $\dot{\phi}_{0}=-\left(a^{3} / 3 \dot{a}\right) V^{\prime}$. This era is supposed to give rise to a reheating period whereby the universe is repopulated with ordinary matter fields, and then to a standard hot big bang cosmology leading up to the present cosmological time. The functional form of $a(\eta)$ during these latter periods is, of course, different but we will ignore such details on the account that most of the change in the value of $a$ occurs during the inflationary regime. The overall normalization of the scale factor will be set so $a=1$ at the "present cosmological time". The inflationary regime would end for a value of $\eta=\eta_{0}$, negative and very small in absolute terms.

The perturbed metric can be written

$$
d s^{2}=a(\eta)^{2}\left[-(1+2 \Psi) d \eta^{2}+(1-2 \Psi) \delta_{i j} d x^{i} d x^{j}\right],
$$

\footnotetext{
${ }^{5}$ We will be using units where $c=1$ but will keep $\hbar$ (with units of Mass $M$ times Length $L$ ), and $G$ ( with units of $L / M$ ) explicitly throughout the manuscript. The coordinates in the metric $\eta, x^{i}$ will have units of length $L$ but the metric components, such as the scale factor $a$ will be dimensionless. The field $\phi$ has units of $(M / L)^{1 / 2}$, and the potential $V$ has units of $M / L^{3}$
} 
where $\Psi$ stands for the relevant perturbation and is called the Newtonian potential.

The perturbation of the scalar field leads to a perturbation of the energy momentum tensor, and thus Einstein's equations at lowest order lead to

$$
\nabla^{2} \Psi=4 \pi G \dot{\phi}_{0} \delta \dot{\phi}=s \delta \dot{\phi}
$$

where we introduced the abbreviation $s=4 \pi G \dot{\phi}_{0}$.

Now we consider the quantum theory of the field $\delta \phi$. It is convenient to work with the rescaled field variable $y=a \delta \phi$ and its conjugate momentum $\pi=\delta \dot{\phi} / a$. To avoid some distracting infrared problems we set the problem in a finite box of side $L$, and decompose the real field and momentum operators as

$$
\hat{y}(\eta, \vec{x})=\frac{1}{L^{3}} \sum_{\vec{k}} e^{\overrightarrow{i k} \cdot \vec{y}_{k}} \hat{y}_{k}(\eta), \quad \hat{\pi}^{(y)}(\eta, \vec{x})=\frac{1}{L^{3}} \sum_{\vec{k}} e^{\overrightarrow{i k} \cdot \vec{x}} \hat{\pi}_{k}(\eta),
$$

where the sum is over the wave vectors $\vec{k}$ satisfying $k_{i} L=2 \pi n_{i}$ for $i=1,2,3$ with $n_{i}$ integer and where $\hat{y}_{k}(\eta) \equiv y_{k}(\eta) \hat{a}_{k}+\bar{y}_{k}(\eta) \hat{a}_{-k}^{\dagger}$ and $\hat{\pi}_{k}(\eta) \equiv g_{k}(\eta) \hat{a}_{k}+\bar{g}_{k}(\eta) \hat{a}_{-k}^{\dagger}$ with

$$
y_{k}^{( \pm)}(\eta)=\frac{1}{\sqrt{2 k}}\left(1 \pm \frac{i}{\eta k}\right) \exp ( \pm i k \eta), \quad g_{k}^{ \pm}(\eta)= \pm i \sqrt{\frac{k}{2}} \exp ( \pm i k \eta) .
$$

As we will be interested in considering a kind of self induced collapse which operates in close analogy with a "measurement", it is convenient to work with Hermitian operators, which in ordinary quantum mechanics are the ones susceptible of direct measurement. Thus we decompose both $\hat{y}_{k}(\eta)$ and $\hat{\pi}_{k}(\eta)$ into their real and imaginary parts $\hat{y}_{k}(\eta)=\hat{y}_{k}{ }^{R}(\eta)+i \hat{y}_{k} I(\eta)$ and $\hat{\pi}_{k}(\eta)=\hat{\pi}_{k}^{R}(\eta)+$ $i \hat{\pi}_{k}^{I}(\eta)$ where

$$
{\hat{y_{k}}}^{R, I}(\eta)=\frac{1}{\sqrt{2}}\left(y_{k}(\eta) \hat{a}_{k}^{R, I}+\bar{y}_{k}(\eta) \hat{a}_{k}^{\dagger R, I}\right), \quad \hat{\pi}_{k}^{R, I}(\eta)=\frac{1}{\sqrt{2}}\left(g_{k}(\eta) \hat{a}_{k}^{R, I}+\bar{g}_{k}(\eta) \hat{a}_{k}^{\dagger R, I}\right) .
$$

We note that the operators $\hat{y}_{k}^{R, I}(\eta)$ and $\hat{\pi}_{k}^{R, I}(\eta)$ are, therefore, hermitian operators. Note that the operators corresponding to $k$ and $-k$ are identical in the real case (and identical, up to a sign, in the imaginary case).

We, now, proceed to calculate the commutator, and we will find out that any of them are standard:

$$
\left[\hat{a}_{k}^{R}, \hat{a}_{k^{\prime}}^{R}\right]=\hbar L^{3}\left(\delta_{k, k^{\prime}}+\delta_{k,-k^{\prime}}\right), \quad\left[\hat{a}_{k}^{I}, \hat{a}_{k^{\prime}}^{I}\right]=\hbar L^{3}\left(\delta_{k, k^{\prime}}-\delta_{k,-k^{\prime}}\right)
$$

Next, we specify the way we will model the collapse, and follow the field evolution through this collapse to the end of inflation. We will assume that the collapse is somehow analogous to an imprecise measurement ${ }^{6}$ of the operators $\hat{y}_{k}^{R, I}(\eta)$ and $\hat{\pi}_{k}^{R, I}(\eta)$ which, as we pointed out are hermitian operators and thus reasonable observables. These field operators contain complete information about the field (we ignore here, for simplicity, the relations between the modes $k$ and $-k$ ).

\footnotetext{
${ }^{6} \mathrm{An}$ imprecise measurement of an observable is one in which one does not end with an exact eigenstate of that observable but rather with a state which is only peaked around the eigenvalue. Thus, we could consider measuring a certain particle's position and momentum so as to end up with a state that is a wave packet with both position and momentum defined to a limited extent, and which, of course, does not entail a conflict with Heisenberg's uncertainty bound.
} 
Let $|\Xi\rangle$ be any state in the Fock space of $\hat{y}$. Let us introduce the following quantity: $d_{k}^{R, I}=\left\langle\hat{a}_{k}^{R, I}\right\rangle_{\Xi}$. Thus, the expectation values of the modes are expressible as

$$
\left\langle\hat{y}_{k}^{R, I}(\eta)\right\rangle_{\Xi}=\sqrt{2} \Re\left(y_{k}(\eta) d_{k}^{R, I}\right), \quad\left\langle\hat{\pi}_{k}^{(y) R, I}(\eta)\right\rangle_{\Xi}=\sqrt{2} \mathfrak{R}\left(g_{k}(\eta) d_{k}^{R, I}\right) .
$$

For the vacuum state $|0\rangle$ we have, of course, $\left\langle\hat{y}_{k}^{R, I}\right\rangle_{0}=0,\left\langle\hat{\pi}_{k}^{(y)}{ }_{R, I}\right\rangle_{0}=0$, while their corresponding uncertainties are

$$
\left(\Delta \hat{y}_{k}^{R, I}\right)_{0}^{2}=(1 / 2)\left|y_{k}\right|^{2}\left(\hbar L^{3}\right), \quad\left(\Delta \hat{\pi}_{k}^{R, I}\right)_{0}^{2}=(1 / 2)\left|g_{k}\right|^{2}\left(\hbar L^{3}\right) .
$$

Now we will specify the rules according to which collapse happens. Again, at this point our criteria will be simplicity and naturalness.

What we have to describe is the state $|\Theta\rangle$ after the collapse. It turns out that, for the goals at hand, all we need to specify $d_{k}^{R, I}=\left\langle\Theta\left|\hat{a}_{k}^{R, I}\right| \Theta\right\rangle$. In the vacuum state, $\hat{y}_{k}$ and $\hat{\pi}_{k}^{(y)}$ individually are distributed according to Gaussian distributions centered at 0 with spread $\left(\Delta \hat{y}_{k}\right)_{0}^{2}$ and $\left(\Delta \hat{\pi}_{k}^{(y)}\right)_{0}^{2}$, respectively. However, since they are mutually non-commuting, their distributions are certainly not independent. In our collapse model, we do not want to distinguish one over the other, so we will ignore the noncommutativity and make the following assumption about the (distribution of) state(s) $|\Theta\rangle$ after collapse:

$$
\begin{gathered}
\left\langle\hat{y}_{k}^{R, I}\left(\eta_{k}^{c}\right)\right\rangle_{\Theta}=x_{k, 1}^{R, I} \sqrt{\left(\Delta \hat{y}_{k}^{R, I}\right)_{0}^{2}}=x_{k, 1}^{R, I}\left|y_{k}\left(\eta_{k}^{c}\right)\right| \sqrt{\hbar L^{3} / 2}, \\
\left\langle\hat{\pi}_{k}^{(y) R, I}\left(\eta_{k}^{c}\right)\right\rangle_{\Theta}=x_{k, 2}^{R, I} \sqrt{\left(\Delta \hat{\pi}_{k}^{(y) R, I}\right)_{0}^{2}}=x_{k, 2}^{R, I}\left|g_{k}\left(\eta_{k}^{c}\right)\right| \sqrt{\hbar L^{3} / 2},
\end{gathered}
$$

where $x_{k, 1}^{R, I}, x_{k, 2}^{R, I}$ are selected randomly from within a Gaussian distribution centered at zero with spread one. Here, we must emphasize that our universe corresponds to a single realization of these random variables, and thus each of these quantities has a single specific value. From the equations above, we solve for $d_{k}^{R, I}$, and using the result in Eq. (5.10) we obtain $\left.\hat{y}_{k} R, I(\eta)\right\rangle$ and $\left\langle\hat{\pi}_{k}^{(y)} R, I(\eta)\right\rangle$ for the state that resulted from the collapse for all later times.

We should keep in mind that this specific recipe for the collapse is just an example among the simple and natural ones, and that other possibilities do exist, and those may lead to different predictions. In fact, in (1) an alternative recipe was considered, that seems to be quite promising in dealing with the fine tuning problem that generically affect inflationary models (23).

\section{Analysis of the Phenomenology}

Now, we must put together our semi-classical description of of the gravitational D.O.F. and the quantum mechanics description of the inflaton field. We recall that this entails the semi-classical version of the perturbed Einstein's equation that, in our case, reduces to:

$$
\nabla^{2} \Psi=s\langle\delta \dot{\phi}\rangle=(s / a)\left\langle\hat{\pi}^{(y)}\right\rangle
$$

The Fourier components at the conformal time $\eta$ are given by:

$$
\Psi_{k}(\eta)=-\left(s / a k^{2}\right)\left\langle\hat{\pi}_{k}^{(y)}(\eta)\right\rangle
$$


Where the expectation value is the one corresponding to the appropriate state of the quantum field. Thus, before the collapse, the state is the vacuum and, therefore, $\Psi_{k}(\eta)=0$, and the space-time is still homogeneous and isotropic at the corresponding scale, while after the collapse we have

$$
\Psi_{k}(\eta)=-\left(s / a k^{2}\right)\left\langle\hat{\pi}_{k}^{(y)}(\eta)\right\rangle_{\Theta}=-\left(s / a k^{2}\right)\left[\left\langle\hat{\pi}_{k}^{(y) R}(\eta)\right\rangle_{\Theta}+i\left\langle\hat{\pi}_{k}^{(y) I}(\eta)\right\rangle_{\Theta}\right]
$$

which no longer vanishes, indicating that after this time the universe became anisotropic and inhomogeneous at the corresponding scale. We now can reconstruct the space-time value of the Newtonian potential using

$$
\Psi(\eta, \vec{x})=\frac{1}{L^{3}} \sum_{\vec{k}} e^{i \vec{k} \cdot \vec{x}} \Psi_{k}(\eta),
$$

which can be used to extract the quantities of observational interest. The measured quantity is essentially, through its imprint on the temperature fluctuations, the "Newtonian potential" on the surface of last scattering: $\Psi\left(\eta_{D}, \vec{x}_{D}\right)$. This quantity is identified with the temperature fluctuations on the surface of last scattering, by regarding those as due to the gravitational red shift associated with the gravitational potential well from which the photons emerge ${ }^{7}$. From this quantity, one extracts

$$
\alpha_{l m}=\int \Psi\left(\eta_{D}, \vec{x}_{D}\right) Y_{l m}^{*} d^{2} \Omega
$$

To evaluate the expected value for the quantity of interest we use (6.3) and (6.4) to write

$$
\Psi(\eta, \vec{x})=\sum_{\vec{k}} \frac{s U(k)}{k^{2}} \sqrt{\frac{\hbar k}{L^{3}}} \frac{1}{2 a} F(\vec{k}) e^{i \vec{k} \cdot \vec{x}},
$$

where the factor $U(k)$ represents the aspects of the evolution of the quantity of interest associated with the physical processes occurring during the period from re-heating to decoupling, which include, among others, the acoustic oscillations of the plasma and which are not central to the issues we are exploring in this work.

Then, after some algebra one finds

$$
\alpha_{l m}=s \sqrt{\frac{\hbar}{L^{3}}} \frac{1}{2 a} \sum_{\vec{k}} \frac{U(k) \sqrt{k}}{k^{2}} F(\vec{k}) 4 \pi i^{l} j_{l}\left(\left(|\vec{k}| R_{D}\right) Y_{l m}(\hat{k}),\right.
$$

where $j_{l}(x)$ is the spherical Bessel function of the first kind, and where $\hat{k}$ indicates the direction of the vector $\vec{k}$. It is in this expression that the justification for the use of statistics becomes clear. The quantity we are in fact considering is the result of the combined contributions of an ensemble of harmonic oscillators, each one contributing with a complex number to the sum, leading to what is, in effect, a two dimensional random walk whose total displacement corresponds to the observational quantity. To proceed further, we must evaluate the most likely value for such total displacement. This we do with the help of the imaginary ensemble of universes, and the identification of the most likely value with the ensemble mean value. Note that this is used here only as a

\footnotetext{
${ }^{7}$ The gravitational change in frequency $\Delta(v) / v=\Psi$, leads through the black body radiation formula to $\Delta(v) / v=$ $\Delta(T) / T$ and therefore $\Delta(T) / T=\Psi$.
} 
calculational tool to extract the most likely value of the "random walk". No other role is given to the ensemble and no call for the existence of its elements is invoked. Now we compute the expected magnitude of this quantity. Taking the continuum limit, and after some algebra (1) we find,

$$
\left|\alpha_{l m}\right|_{M . L .}^{2}=\frac{s^{2} \hbar}{2 \pi a^{2}} \int \frac{U(k)^{2} C(k)}{k^{4}} j_{l}^{2}\left(\left(|\vec{k}| R_{D}\right) k^{3} d k\right.
$$

where some of the information contained in $F(k)$ has become encoded in:

$$
C(k) \equiv 1+\left(2 / z_{k}^{2}\right) \sin \left(\Delta_{k}\right)^{2}+\left(1 / z_{k}\right) \sin \left(2 \Delta_{k}\right)
$$

where $\Delta_{k}=k \eta-z_{k}, z_{k}=\eta_{k}^{c} k$ with $\eta$ represents the conformal time of observation, and $\eta_{k}^{c}$ the conformal time of collapse of the mode $k$.

The last expression for $\left|\alpha_{l m}\right|_{M . L .}^{2}$ can be made more useful by changing the variables of integration to $x=k R_{D}$ leading to

$$
\left|\alpha_{l m}\right|_{M . L .}^{2}=\frac{s^{2} \hbar}{2 \pi a^{2}} \int \frac{U\left(x / R_{D}\right)^{2} C\left(x / R_{D}\right)}{x^{4}} j_{l}^{2}(x) x^{3} d x,
$$

This expression reveals that, if one ignores late time physics processes represented in $U$ and the remaining signatures of the collapse process represented in $C$, the observational spectrum should have no dependence on the size of the surface of last scattering $R_{D}$.

Turning our attention to the expression in Eq. (6.9), we note that the appearance of the time of observation could, in principle, lead to concerns, as that should be taken as the time of decoupling, which is thought to lie in a regime where inflation has long ended. In practice, its effects can be expected to be negligible because (in our convention) that conformal time would be a an exponentially small negative number (i.e. $\eta \rightarrow 0^{-}$as $a \rightarrow \infty$ ). Next we note, in order to get a reasonable spectrum, there seems to be only one simple option: that $z_{k}$ is essentially independent of $k$, that is, the time of collapse of the different modes should depend on the mode's frequency according to $\eta_{k}^{c}=z / k$. In fact, the standard answer, in the absence of late time physical effects such as plasma oscillations, would correspond to $C(k)=1$. This is a rather strong conclusion which could represent relevant information about whatever the mechanism of collapse is, and leads, as we will see, to actual tests on the feasibility of different mechanisms for the physical collapse.

To end this section, we consider a question raised during the talk, by W. Unruh. He asked about the reason behind the fact that the oscillatory behavior reflected in $C(k)$ appears in the present analysis and not in the standard treatments, despite the fact that the system is linear and linear averages can not be expected to hide any real feature. To address this issue, going beyond the calculation itself requires a simple analogy. I believe that it is provided by the consideration of a simple harmonic oscillator initially prepared on a highly excited eigen-state of the hamiltonian. If all our treatment focusses on carrying averages of quantities extracted directly from the initial state we will find no trace of any oscillatory behavior in time. However, if we consider the state resulting of a partial or inaccurate measurement of the position, which is considered to be naturally described by a coherent state, the time of the measurement or collapse of the initial state into the coherent state will be of relevance and from that point onwards oscillations in time can be expected for various of the relevant physical quantities. 


\section{A version of 'Penrose's mechanism' for collapse in the cosmological setting}

Penrose has advocated, for a long time, that the collapse of quantum mechanical wave functions might be a dynamical process independent of observation, and that the underlying mechanism might be related to gravitational interaction. More precisely, according to this suggestion, collapse into one of two quantum mechanical alternatives would take place when the gravitational interaction energy between the alternatives exceeds a certain threshold. In fact, much of the initial motivation for the present work came from Penrose's ideas and his questions regarding the quantum history of the universe.

A very naive realization of Penrose's ideas in the present setting could be obtained as follows: each mode would collapse by the action of the gravitational interaction between its own possible realizations. In our case, one could estimate the interaction energy $E_{I}(k, \eta)$ by considering two representatives of the possible collapsed states on opposite sides of the Gaussian associated with the vacuum. We will denote the two alternatives by the indices (1) and (2). Clearly, we must interpret $\Psi$ as the Newtonian potential and, consequently, the right hand side of Eq. (5.5), (after a rescaling by $a^{-2}$ to replace the laplacian expressed in the comoving coordinates $x$ to a laplacian associated with coordinates measuring physical length ) should be identified with matter density $\rho$. Therefore, $\rho=a^{-2} \dot{\phi}_{0} \dot{\delta} \phi=a^{-3} \dot{\phi}_{0} \pi^{y}$. Then the relevant energy is given by :

$$
E_{I}(\eta)=\int \Psi^{(1)} \rho^{(2)} d V=\int \Psi^{(1)}(x, \eta) \rho^{(2)}(x, \eta) a^{3} d^{3} x=\int \Psi^{(1)}(x, \eta) \dot{\phi}_{0}\left(\pi^{y}(x, \eta)\right)^{(2)} d^{3} x .
$$

where $\Psi^{(1)}$ represents the Newtonian potential that would have arisen if the system had collapsed into the alternative (1), and $\rho^{(2)}$ represents the density perturbation associated with a collapse into the alternative (2). Note that in this section we are ignoring the overall sign of this energy which being a gravitational binding energy would naturally be negative. We next express this energy in terms of the Fourier expansion leading to :

$$
E(\eta)=\left(1 / L^{6}\right) \Sigma_{k, k^{\prime}} \Psi_{k}^{(1)}(\eta) \dot{\phi}_{0}\left(\pi^{y}\right)_{k^{\prime}}^{(2)}(\eta) \int e^{i x\left(k-k^{\prime}\right)} d^{3} x=\left(1 / L^{3}\right) \dot{\phi}_{0} \Sigma_{k} \Psi_{k}^{(1)}(\eta)\left(\pi^{y}\right)_{k}^{(2)}(\eta),
$$

where (1), (2) refer to the two different realizations chosen. Recalling that $\Psi_{k}=\left(s / a k^{2}\right) \pi_{k}^{y}$, with $s=4 \pi G \dot{\phi}_{0}$, we find

$$
E(\eta)=4 \pi G\left(a / L^{3}\right) \dot{\phi}_{0}^{2} \Sigma_{k}\left(1 /(a k)^{2}\right)\left(\pi^{y}\right)_{k}^{(1)}(\eta)\left(\pi^{y}\right)_{k}^{(2)}(\eta),
$$

Using equation (5.11), we estimate $\left(\pi_{k}^{y}\right)^{(1)}(\eta)\left(\pi_{k}^{y}\right)^{(2)}(\eta)$ by $\left|<\pi_{k}^{y}>\right|^{2}=\hbar k L^{3} / 4$, and thus we obtain:

$$
E_{I}(\eta)=\Sigma_{k}(\pi \hbar G / a k)\left(\dot{\phi}_{0}\right)^{2} .
$$

which can be interpreted as the sum of the contributions of each mode to the interaction energy of different alternatives. According to all the considerations we have made, we view each mode's collapse as occurring independently, so the trigger for the collapse of mode $k$ would be, in accordance to the scheme based on Penrose's ideas, the condition that this energy $E_{I}(k, \eta)=(\pi \hbar G / a k)\left(\dot{\phi}_{0}\right)^{2}$ reaches the 'one-graviton level', namely, that it equals the value of the Planck Mass $M_{p}$. Now we 
use the specific expressions for the scale factor $a=\frac{-1}{\eta H_{I}}$ and the slow rolling of the background scalar field $\dot{\phi}_{0}=-(1 / 3)\left(a^{3} / \dot{a}\right) V^{\prime}$ to find

$$
E_{I}(k, \eta)=\frac{\pi \hbar G}{9 H_{I}^{2}}(a / k)\left(V^{\prime}\right)^{2}
$$

Thus, the condition determining the time of collapse $\eta_{k}^{c}$ of the mode $k$ is that the above expression reaches the value $M_{p}$. Thus, we find:

$$
z_{k}=\eta_{k}^{c} k=\frac{\pi}{9}\left(\hbar V^{\prime}\right)^{2}\left(H_{I} M_{p}\right)^{-3}=\frac{\varepsilon}{8 \sqrt{6 \pi}}(\tilde{V})^{1 / 2} \equiv z^{c}
$$

which is independent of $k$, and, thus, leads to a roughly scale invariant spectrum of fluctuations in accordance with observations. It is worth pointing out that this result is far from trivial or expected on simple dimensional grounds, as the dimensionless factor $a$ could conceivable have appeared with the incorrect power in Eq.( 7.5). Moreover, we note that, as the energy of mode $k$ during the slow roll regime is, as shown in Eq. (7.5), an increasing function of conformal time $\eta$, at the very early times the condition for collapse would not have been be fulfilled, and it is only as the universe expands that such point would be attained.

One question that was raised during the presentation of this talk by T. Jacobson, concerns the finiteness of energy created during each collapse and its independence of the "size" of the universe. In this regard, we note that the calculation of the energies above is analogous to the calculation of the vacuum energy of a quantum field in Minkowski space-time, in its ground state: as each mode of the quantum field is essentially an harmonic oscillator its vacuum energy, regardless of the infinitude of the universe, is a finite quantity : $(1 / 2) \hbar \omega$. Similarly, in our case, the energy created in the collapse of each mode is finite. However, and in complete analogy with the standard quantum field considerations, the sum over all modes gives infinite if the number of modes is infinite. This is in both, the present case and the ordinary quantum field theory case, an ultraviolet problem and has no relation with the universe's "size".

Note that the formalism allows us to look closer into the collapse issue and to ask for instance: when do the relevant modes collapse? In order to answer this question we use the value for $z^{c}$ and recall that the time of collapse is determined by $\eta_{k}^{c}=z^{c} / k$, and thus the scale factor at the time of collapse of the modes with wave number $k$ was

$$
a_{k}^{c}=\left(H_{I} \eta_{k}^{c}\right)^{-1}=(12 / \varepsilon) k l_{p}(\tilde{V})^{-1}
$$

where $l_{p}$ stands for the Planck length, and $\varepsilon \equiv(1 / 2)\left(M_{P l}^{2} / \hbar\right)\left(V^{\prime} / V\right)^{2}$ is the so called slow roll parameter of the inflationary model. As the value of the scale factor $a$ at the last scattering surface was $a \approx 10^{-4}$ (recall that the scale factor $a$ has been set so its value today is 1 ), the modes that are relevant to say scales of order $10^{-3}$ of the size of the surface of last scattering (corresponding to a fraction of a degree in today's sky) have $k \approx 10^{-10} l y^{-1}$.

Thus, taking $\varepsilon \times \tilde{V}$ to be of order $10^{-5}$, we have for those modes $a_{k}^{c} \approx 10^{-45}$ corresponding to $N_{e}=103$ e-folds of total expansion, or something like 80 e-folds before the end of inflation in standard type of inflationary scenarios. Thus, in this scheme inflation must have at least $90 \mathrm{e}$-folds for it to include the complete description of the regime we are considering and to account also for 
the collapse of the modes that are of the order of magnitude of the surface of last scattering itself. The usual requirements of inflation put the lowest bound at something like 60 e-folds of inflation so the present requirement is not substantially stronger.

This result can be directly compared with the so called, time of "horizon crossing" $\eta_{k}^{H}$ for mode $k$, corresponding to the physical wavelength reaching the Hubble distance $H_{I}^{-1}$. Therefore this latter time is determined from:

$$
a_{k}^{H} \equiv a\left(\eta_{k}^{H}\right)=k /\left(2 \pi H_{I}\right)=k l_{p}\left(3 / 32 \pi^{3}\right)^{1 / 2}(\tilde{V})^{-1 / 2} .
$$

Thus, the ratio of scale factors at collapse and at horizon crossing for a given mode is $a_{k}^{c} / a_{k}^{H}=$ $(16 / \varepsilon)\left(6 \pi^{3}\right)^{1 / 2}(\tilde{V})^{-1 / 2}$, which would ordinarily be a very large number, indicating that the collapse time would be much later than the time of "horizon exiting" or crossing out, of the corresponding mode.

Thus, we find that a naive realization of Penrose's ideas seems, at first sight, to be a good candidate to supply the element that we argued is missing in the standard accounts of the emergence of the seeds of cosmic structure from quantum fluctuations during the inflationary regime in the early universe. However, more research along these lines is necessary to find out, for instance, whether the scheme would imply a second collapse of modes already collapsed, and whether such secondary collapse could disrupt in a substantial way the observational spectrum.

\section{Predictions and discussion}

It is quite clear that it would be very hard to find a scheme in which the function $C(k)$ would be exactly a constant, and that some dependence of $k$ will remain in any reasonable collapse scheme. A particularly robust source of this effect is associated with the finite time of decoupling as can be seen in in the expression for the function $C(k)$ 6.9. These dependences will, in turn, lead to slight deviations from the standard form of the spectrum before the inclusion of the late time physics such as the plasma oscillations. This, in turn, can be expected to leave some traces in the observational CMB spectrum that could, conceivable, be searched for observationally.

However, the most striking prediction of the scheme, is the absence of tensor modes, or at least their very strong suppression. The reason for this can be understood by considering the semiclassical version of Einstein's equation and its role in describing the manner in which the inhomogeneities and anisotropies in the metric arise in our scheme. As indicated in the introduction, the metric is taken to be an effective description of the gravitational D.O.F., in the classical regime, and not as the fundamental D.O.F. susceptible to be described at the quantum level. It is thus the matter degrees of freedom (which in the present context are represented by the inflaton field) the ones that are described quantum mechanically and which, as a result of a fundamental aspect of gravitation at the quantum level, undergo effective quantum collapse (the reader should recall that our point of view is that gravitation at the quantum level will be drastically different from standard quantum theories, and that, in particular, it will not involve universal unitary evolution). This leads to a nontrivial value for $\left\langle T_{\mu v}\right\rangle$, which leads to the appearance of the metric fluctuations. The point is that the energy momentum tensor contains linear and quadratic terms in the expectation values of the quantum matter field fluctuations, which are the source terms determining the geometric 
perturbations. And a close examination reveals that, in the case of the scalar perturbations, we have first order contributions proportional to $\dot{\phi}_{0}\langle\delta \dot{\phi}\rangle$ while no similar first order terms appear as source of the tensor perturbations ( i.e. of the gravitational waves). At the time of the writing of this article, the tensor modes had not been detected, in contrast with the scalar modes, a fact that seems to lead support to the prediction above.

Next we consider briefly the relationship between the present analysis and the programs that search for a quantum theory of gravity. The first thing to note is that there is, in principle, no intrinsic conflict between the present analysis with any program that contemplates that the fundamental degrees of freedom are not necessarily directly tied with the observable space-time geometry. On the other hand, a conflict could arise if the quantum gravity theory required the geometric D.O.F. to be quantized on equal footing and in all circumstances where the matter degrees of freedom would require a quantum description, leading to a situation that would invalidate the applicability in the present, and similar contexts, of the semi-classical Einstein's equations. The challenge for a theory of quantum gravity that is posed by the present analysis is to provide an characterization of the circumstances in which that semiclassical approach would be justified (which would include the cosmological situation at hand ) while at the same time provide an explanation for the effective mechanism of collapse that we are attributing to some unknown aspect of quantum gravity.

The search for manifestations of quantum aspects of gravitation, after being practically ignored for a long time, has transformed, as of late, into such an attractive enterprise that a large number of researchers have become strongly attracted even by some ideas of doubtful consistency and unclear interpretation. It seems that in this "Gold Rush", at least some very interesting and direct avenues have been ignored. The case of the emergence of the seeds of cosmic structure, the only known process, proved to be observationally accessible, in which gravity and quantum physics seem to be inexorably tied, seems to be the most glaring example. On the other hand, it is naturally quite surprising, at least within the quantum gravity community, that, something that started as what could be called "purely philosophical considerations", would lead to analysis that can be directly confronted with observations, and which give rise to predictions that could, in principle, invalidate aspects of the emergent proposals. The lesson we draw from this case is that in the search for clues of aspects of quantum gravity one has to face the most obscure issues head on rather than ignore them. This is, of course true, in any scientific enterprise but it is more so in a field such a quantum gravity, where there are so few pointers beyond the need of consistency as one of the few reliable guiding principles.

\section{Acknowledgments}

I wish to thank the conference organizers for the hospitality. This work was supported in part by the grant 43914-F of CONACyT .

\section{References}

[1] "On the Quantum Origin of the Seeds of Cosmic Structure" A. Perez, H. Sahlmann, and D. Sudarsky, Classical and Quantum Gravity 23, 2317, (2006) [arXiv: gr-qc/0508100]. 
[2] "Quantum Mechanics of the scalar field in the new inflationary Universe", A. Guth and S.-Y. Pi Phys. Rev. D 32, 1899, (1985); "Fluctuations in the Inflationary Universe", S. W. Hawking Nucl. Phys. B 224, 180, (1983); "Origin of Structure in the Universe" J.J. Halliwell and S. W. Hawking, Phys. Rev. D, 31, 1777, (1985).

[3] "Cosmological parameters From First results of Boomerang" A.E. Lange et. al. Phys. Rev. D,63, 042001, (2001); G. Hinshaw et. al., Astrophys. J. Supp., 148, 135, (2003); "Power Spectrum of Primordial Inhomogeneity Determined from four Year COBE DMR SKY Maps", K.M. Gorski et. al. Astrophys. J. 464, L11, (1996); "First Year Wilkinson Micorowave Anisotropy Probe (WMAP) Observations: Preliminary Results" C.L. Bennett et. al.Astrophys. J. Suppl. 148, 1, (2003); "First Year Wilkinson Micorwave Anisotropy Probe (WMAP) Observations: Foreground Emission", C. Bennett et al. Astrophys. J. Suppl. 148, 97, (2003).

[4] For reviews abut the various approaches to the measurement problem in quantum mechanics see for instance the classical reference "Philosophy of quantum mechanics. The interpretations of quantum mechanics in historical perspective" M. Jammer, ( John Wiley and Sons, New York 1974); or the more recent "Quantum Theory: Concepts and Methods", A. Peres (Kluwer, Academic Publishers, 1993). "The Interpretation of Quantum Mechanics" R. Omnes, ( Princeton University Press 1994), and the more specific critiques "Why Decoherence has not Solved the Measurement Problem: A Response to PW Anderson" - S. L. Adler Stud. Hist. Philos. Mod. Phys. 34135-142 (2003), [arXiv: quant-ph/0112095]ã Why ÒmodalÓ interpretations of quantum mechanics don't solve the measurement problem A Elby, Found. of Phys. Lett. 6, 5-19 (1993) and the review of approaches to the problem presented in A. Bassi G. C. Ghirardi, Phys. Rept ,379, 257, (2003)[arXiv: quant-ph/0302164];

[5] “The Emperor's New Mind", R. Penrose, The Emperor's New Mind, (Oxford University Press 1989); R. Penrose, On Gravity's Role in Quantum State Reduction, in Physics meets philosophy at the Planck scale Callender, C. (ed.) (2001).

[6] "Quantum Cosmology Problems for the $21^{\text {st }}$ Century", J. B. Hartle, [arXiv: gr-qc/9701022]; "Generalized Quantum mechanics for Quantum Gravity", J. B. Hartle, [arXiv: grqc/0510126].

[7] "Fluctuations at the threshold of classical cosmology" E. R. Harrison, Phys. Rev. D, 1, 2726, (1970); Y. B. Zel'dovich Mon. Not. Roy. Astron. Soc. 160, 1p (1972).

[8] "A Unified Dynamics For Micro And Macro Systems", G. C. Ghirardi, A. Rimini, and T. Weber,Phys. Rev. D 34, 470, (1986).

[9] Arnold Neumaier, in the "theoretical physics FAQ" at his University of Wien, webpage: http://www.mat.univie.ac.at/ neum/physics-faq.txt.

[10] "Decoherence, the measurement problem and interpretations of quantum Mechanics" $M$. Schlosshauer, Rev. Mod. Phys. 76, 1267 (2004), [arXiv: quant-ph/0312059, page 9]. 
[11] E. Joos, in "Elements of Environmental Decoherence", proceedings of the conference Decoherence Theoretical, Experimental and Conceptual Problems" Eds. P. Blanchard, D. Giulini, E. Joos, C. Keifer and I. O. Stamatescu ( Springer 1999) [arXiv: quant-ph/9908008].

[12] "Decoherence in Quantum Cosmology", J.J. Halliwell, Phys. Rev. D, 39, 2912,(1989); “Origin of Classical Structure From Inflation", C. Kiefer Nucl. Phys. Proc. Suppl. 88, 255 (2000) [arXiv:astro-ph/0006252]; "Semiclassicality and decoherence of Cosmological perturbations", D. Polarski and A.A. Starobinsky,Class. Quant. Grav. 13, 377 (1996) [arXiv: grqc/9504030]; “ Environment Induced Superselection In Cosmology", W.H. Zurek, Environment Induced Superselection In Cosmology in Moscow 1990, Proceedings, Quantum gravity (QC178:S4:1990), p. 456-472. (see High Energy Physics Index 30 (1992) No. 624); “Gauge Invariant Cosmological Perturbations" R. Branderberger H. Feldman and V. Mukhavov,Phys. Rep. 215, 203, (1992); "Decoherence Funtional and Inhomogeneities in the Early Universe", R. Laflamme and A. Matacz Int. J. Mod. Phys. D 2, 171 (1993) [arXiv:gr-qc/9303036]; "The self-induced approach to decoherence in cosmology," M. Castagnino and O. Lombardi, Int. J. Theor. Phys. 42, 1281, (2003), [arXiv:quant-ph/0211163]; "Decoherence during inflation: The generation of classical inhomogeneities," F. C. Lombardo and D. Lopez Nacir, Phys. Rev. D 72, 063506 (2005) [arXiv:gr-qc/0506051]; "Inflationary Cosmological Perturbations of Quantum Mechanical Origin" J. Martin, Lect. Notes Phys. 669, 199 (2005) [arXiv:hepth/0406011]; " Best Unbiased Estimates for Microwave background Anisotropies", L.P. Grishchuk and J. Martin, Phys. Rev. D 56, 1924 (1997) [arXiv:gr-qc/9702018]; "Decoherence in Quantum Cosmology at the onset of Inflation", A.O. Barvinsky, A.Y. Kamenshchik, C. Kiefer, and I.V. Mishakov, Nucl. Phys. B 551, 374 (1999) [arXiv:gr-qc/9812043];

[13] "Quantum To Classical Transition of Cosmological Perturbations for Non Vacuum Initial States", J. Lesggourges, David Polarski and Alexei A. Starobinsky [arXiv: grqc/961101904030].

[14] "Speakable and Unspeakable in Quantum Mechanics", J. S. Bell (Cambridge University Press 1987).

[15] "A New violation of Bell's inequalities" A. Aspect, P. Grangier, G. Roger, Phys. Rev. Lett. 49, 9, (1982).

[16] "Cosmology and Astrophysics Through Problems", T. Padmanabhan (Cambridge University Press 1996).

[17] "The Road to Reality", R. Penrose ( Ed. Alfred A. Kopf- New York, 2006).

[18] B. D’Espagnat, Phys. Lett. A 282, 133, (2000).

[19] "Inflationary perturbations: The Cosmological Schwinger effect" J. Martin, [arXiv: hepth/07043540].

[20] "On Gauge Invariance and Vacuum Polarization" J. Schwinger Phys. Rev. 82, 664, (1951). 
[21] See for instance discussions about the EPR experiment in A. Peres " Quantum Theory: Concepts and Methods" (Kluwer Academic Publishers, 1993)

[22] "Is the Moon There when nobody Looks?" D. Mermin Physics Today 32, 38, (1985).

[23] "The Seeds of Cosmic structure as a door to New Physics" D. Sudarsky, J. Phys. Conf. Ser. 68, 012029, (2007) [arXiv: gr-qc/0612005] . 